Gregor Lämmel, Dominik Grether, Kai Nagel

\title{
The representation and implementation of time-dependent inundation in large-scale microscopic evacuation simulations
}

Journal article | Accepted manuscript (Postprint)

This version is available at https://doi.org/10.14279/depositonce-7730

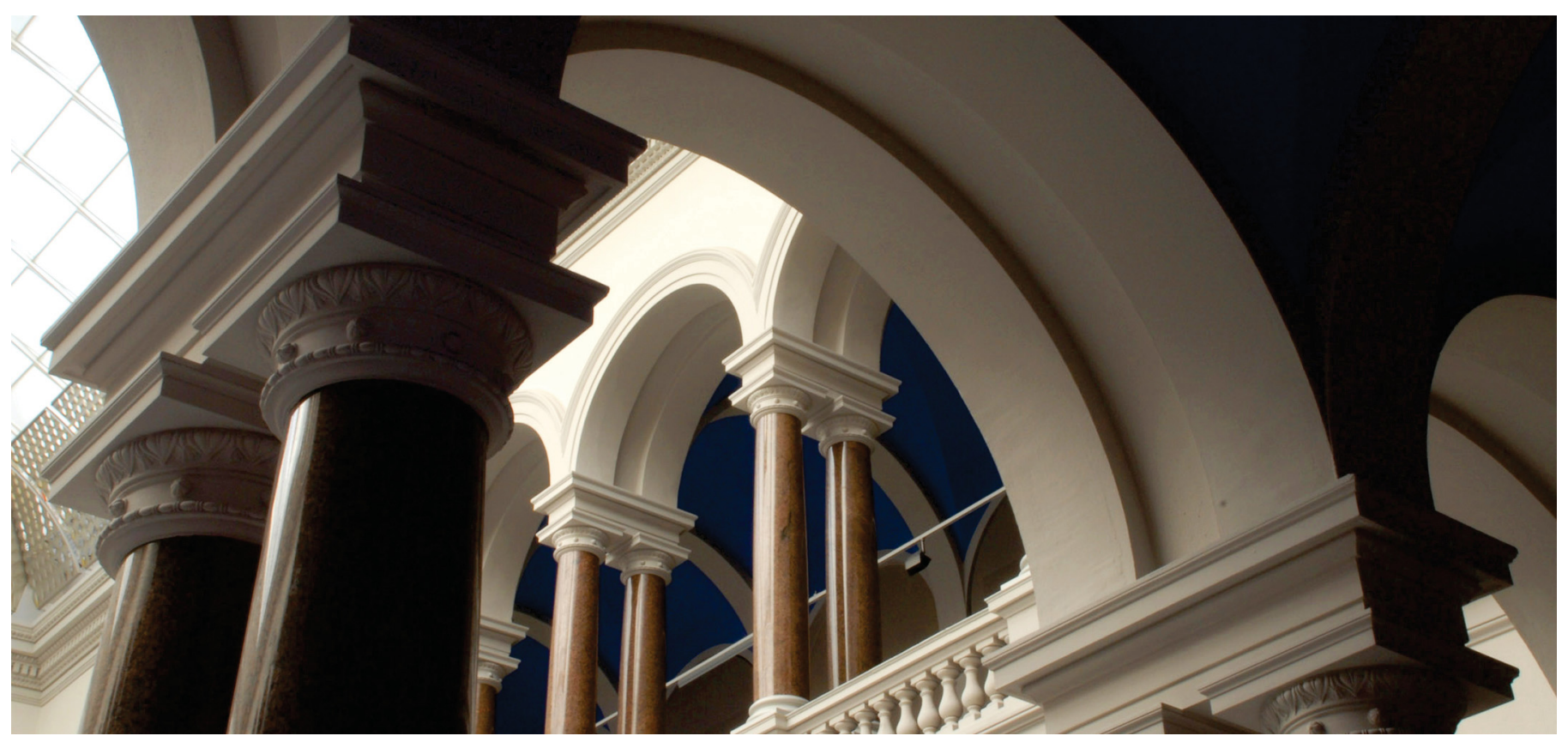

Lämmel, G., Grether, D., \& Nagel, K. (2010). The representation and implementation of time-dependent inundation in large-scale microscopic evacuation simulations. Transportation Research Part C: Emerging Technologies, 18(1), 84-98. https://doi.org/10.1016/j.trc.2009.04.020 


\title{
The representation and implementation of time-dependent inundation in large-scale microscopic evacuation simulations
}

\author{
Gregor Lämmel*, Dominik Grether, Kai Nagel \\ Transport Systems Planning and Transport Telematics, Berlin Institute of Technology, \\ 10587 Berlin, Germany
}

\begin{abstract}
Multi Agent Simulation has increasingly been used for transportation simulation in recent years. With current techniques, it is possible to simulate systems consisting of several million agents. Such Multi Agent Simulations have been applied to whole cities and even large regions. In this paper it is demonstrated how to adapt an existing multi agent transportation simulation framework to large-scale pedestrian evacuation simulation. The underlying flow model simulates the traffic based on a simple queue model where only free speed, bottleneck capacities, and space constraints are taken into account. The queue simulation, albeit simple, captures the most important aspects of evacuations such as the congestion effects of bottlenecks and the time needed to evacuate the endangered area. In the case of an evacuation simulation the network has time dependent attributes. For instance, large-scale inundations or conflagrations do not cover all the endangered area at once.

These time dependent attributes are modeled as network change events. Network change events are modifying link parameters at predefined points in time. The simulation framework is demonstrated through a case study for the Indonesian city of Padang, which faces a high risk of being inundated by a tsunami.
\end{abstract}

Key words: multi agent simulation, large-scale evacuation simulation, time dependent network

\section{Introduction}

The evacuation of whole cities or even regions is an important problem, as demonstrated by recent events such as the evacuation of Houston in the case of Hurricane Rita or the evacuation of coastal cities in the case of tsunamis.

\footnotetext{
*Corresponding author. fon: +49 30 31421376; fax: +493031426269

Email address: laemmel@vsp.tu-berlin.de (Gregor Lämmel)
} 
One example for a highly vulnerable area is the city of Padang, West Sumatra, Indonesia. Sumatra's third largest city is located directly on the coast and partially sited beneath the sea level, and thus is located in a zone of extreme risk due to severe earthquakes and tentatively triggered tsunamis. The city of Padang has been hit by tsunamis in the past. The most well documented tsunamis are the ones from 1797 and 1833 [49]. Both tsunamis inundated large parts of the city. However, these past tsunami events are hardly comparable with the current local situation due to major changes in land use pattern. What is more, population figures have risen strongly. Today the city has approx. $1,000,000$ inhabitants and the most densely populated parts of the city are located directly at the shore line.

The "Last-Mile Evacuation" research project [6, 41] develops a numerical last mile tsunami early warning and evacuation information system on the basis of detailed earth observation data and techniques as well as unsteady,

hydro-numerical modeling of small-scale flooding and inundation dynamics of the tsunami, combined with evacuation simulations in the urban coastal hinterland for the city of Padang.

Since the advance warning time before the tsunami wave reaches the coast line is only 20-40 minutes, the evacuation must be as quick as possible. Even if not all of the estimated 1,000,000 inhabitants need to be evacuated, the number of evacuees could be hundreds of thousands. Therefore a detailed analysis of aspects that could influence the evacuation process is necessary. With this analysis it should be possible to:

- Give an estimate of the evacuation time.

- Detect bottlenecks that could for example emerge at bridges.

- Detect highly endangered areas, where a vertical evacuation ${ }^{1}$ seems the only way.

Because of the complexity of the system, an analytic solution to this problem seems to be hard. Therefore a microscopic multi-agent simulation for the city with all its inhabitants has been developed.

Congruent with the importance of the topic, there is a large body of research regarding emergency evacuations. As a first classification, one may differentiate between two situations: (i) evacuation from within buildings, ships, airplanes, etc.; (ii) large-scale citywide or regional evacuations, e.g. because of nuclear power plants failures or because of hurricanes. Case (i) usually concerns pedestrian evacuation; case (ii) usually uses traffic-based evacuation.

Ongoing research concerns citywide or regional evacuations, i.e. case (ii). The development of these tools was much influenced by the development of tools in the areas of transport planning and traffic management. At the core of many of these methods is a static assignment routine (e.g. [61, 52]). A typical example

\footnotetext{
${ }^{1}$ quake and tsunami proof shelters
} 
for traffic-based evacuation simulation based on static concepts is MASSVAC [29] although later versions contain dynamic aspects.

A shortcoming of static assignment is that it does not possess any consideration of the time-of-day dynamics. In contrast, dynamic traffic assignment (DTA) is defined as a distribution of time-dependent trips on routes. A typical approach to implement DTA is day-to-day re-planning: The traffic flow simulation (also called network loading) is run with pre-specified routes, route costs are extracted, some or all of the routes are modified, the traffic flow simulation is run again, etc., until some stopping criterion is fulfilled. Examples of stopping criteria are that either every trip uses a route which minimizes expected travel time (time-dependent Nash equilibrium), or it selects between different route alternatives following a pre-specified distribution function (time-dependent SUE).

Many DTA packages have been tested in the evacuation context: MITSIM [33], Dynasmart [39, 11], PARAMICS [10], and VISSIM [25]. Oak Ridge National Laboratory has a package named "OREMS" [51] explicitly for evacuation traffic. Publications stressing dynamic aspects of traffic-based evacuation as a novelty can be found with dates as recent as 2000 , e.g. [57, 3]. For a review, see $[2]$.

A good overview of pedestrian evacuation modeling and software can be found in the books of the bi-annual conference series "Pedestrian and Evacuation Dynamics" $[60,16,17,1]$. Pedestrian evacuation simulations can be classified into microscopic and macroscopic ones. Microscopic models represent space, time, and persons on a fine-grained level. Possible microscopic approaches are Cellular Automata (CA) [36], discretized differential equations ("molecular dynamics (MD)") [27, 26], or movement rules based on random utility modelling [4]. Examples of software packages based on microscopic models are Exodus [15], Myriad (www.crowddynamics.com), Egress (www.aeat-safetyand-risk.com/html/egress.html), and PedGo [35]. Macroscopic models use the analogy of flows of pedestrians and liquids. Examples of software packages based on macroscopic models are Aseri [59] and Simulex (www.iesve.com). See Refs. [32] and [38] for surveys. Compared to what is known in terms of field measurements (e.g. [55, 68]), most if not all packages lead to similar results [56].

A large body of work (e.g. $[67,43])$ uses microsimulation to investigate the issues of contraflow evacuation, i.e. the reversal of inbound lanes of a freeway in order to obtain additional outbound capacity. In contrast, little work seems to exist that specifically deals with evacuation using other modes of transport than walking for the evacuation of confined spaces, or driving for the evacuation of cities or regions. Ref. [24] describes mixed evacuation traffic, geared to Taiwanese requirements.

Once the movement model is selected, it is necessary to define the evacuation directions. For more complex geometries, this is no longer a single movement towards one or two exits, but may involve rather complex movments in a building or in a street network. The arguably simplest solution is a grid-based potential function where the "uphill direction" leads to the nearest exit [50]. The same can be done using essentially continous spatial variables, at the expense of much larger computing times [30]. Alternatively, routing can be done along graphs 
$[23,20]$, which is a much faster technique when the abstraction to a graph is possible.

A further distinction is if travelers can re-route while they are on their way (within-day re-planning; en-route re-planning), or only before their trip (day-today re-planning; pre-trip re-planning) [8]. Clearly, en-route re-planning capability is more realistic. It is, however, also more demanding: Adaptation of the plans needs to be called frequently from within the network loading, rather than only having to alternate between the network loading and the mental layers as one does in day-to-day re-planning.

A recent development regarding pedestrian evacuation is that more behavioral aspects are added. For example, people may discard the warning as irrelevant; they may not head for the nearest exit; people have a tendency to follow each other (herding) $[28,42]$. This is matched by a recent tendency, often by agent-oriented software engineering groups, to make the simulated agents more complex in order to include these behavioral aspects $[48,54]$. There seems to be universal agreement that these behavioral aspects increase evacuation times; simulations with reduced behavioral aspects can, in consequence, still be used as an optimistic benchmark.

To our knowledge, none of the above approaches is able to simulate largescale scenarios (with millions of entities) while remaining microscopic: With a CA model, an area of $40 \mathrm{~km} \times 40 \mathrm{~km}$ translates into $10^{10}$ cells. Even if every cell only needs 1 Byte, this still translates into 10 GByte of memory, resulting in large simulation times. For the MD approach, the problem are the sub-second time resolutions that are typically used [14]. DTA approaches seem the most likely candidates, but to our knowledge their implementation of the traffic flow dynamics usually is still too time-consuming for scenarios of that size:

Ref. [58] reports a study using Dynasmart-P consisting of 1347 nodes and 3004 links. 200,000 vehicles were loaded onto the network. The runtime for about 30 iterations of 2 hours of simulation was almost 8 hours. This means running one iteration with this 1347 nodes/ 3004 links scenario takes about 16 minutes. If the runtime scales with the scenario size it would be very time consuming to run larger scenarios. In Ref. [69], the DynaMIT framework was applied to a real-time scenario but on a small network (243 nodes and 606 links). In that study a rolling horizon approach was chosen to have a 5 min estimation and 30 min prediction on that network. Two iterations of estimation and two iterations of prediction took about $1 \mathrm{~min}$. If the runtime scales with the size of the network the performance is comparable to the Dynasmart-P approach and again too slow for large-scale scenarios.

One way to achieve faster computation with a microscopic model is to use a model with deliberately large time steps and to computationally concentrate on those areas (links) where the pedestrian movement actually takes place [21]. Another approach is based up on a modified queuing model $[18,62]$. The queuing model simplifies streets to edges and crossings to nodes; the difference to standard queuing theory is that agents (particles) are not dropped but spill back, causing congestion. This graph-oriented model is defined by free speed, flow capacity, and storage capacity of the edges. This simplification leads to a 
major speedup of the simulation while keeping results realistic. The combination of these two approaches (switching off unused links; queue model) is used in this paper, and leads to computing speeds of about 2 min per iteration for 320000 agents on 17000 links.

Additional speed-ups could be gained by parallel computing [9]. On distributed architectures, however, it is necessary to serialize/de-serialize objects that move from one CPU to another. When done automatically (e.g. [63]), we have always found that this is rather slow and thus only suitable for scenarios where there is little communication compared to the communication; when done manually, it causes considerable software engineering overhead across the whole MATSim project. Shared-memory architectures were in the past quite expensive, but the recent emergence of multi-core architectures and GPUs (graphical processing units) may be attractive options. For first steps in these directions in the context of transportation/pedestrian projects, see matsim.org/node/238 or [64].

In the case of an evacuation simulation the network has time dependent attributes. For instance, large-scale inundations or conflagrations do not cover all the endangered area at once. One solution is to model this as a time dependent network. Time dependent networks have been applied to evacuation planning [44] and are often modeled as time expanded graphs [34, 53, 37]. In a time expanded graph every node/edge is replicated for every time step $t=0,1, \ldots, T$, and additional links are connecting the nodes between the time steps. In contrast, so-called time-aggregated graphs [19] omit the explicit time expansion, and rather use a time-dependent look-up of the link cost. The notation of that reference implies $T$ time intervals which apply uniformly to all links; their $O(\log T)$ time complexity of the link cost lookup implies that these time intervals need not to be equally spaced.

Yet, in the case of a tsunami inundation, the structure of the link cost changes is quite different: There is, at least in an abstract interpretation, only a switch from "passable" to "non-passable", but that switch can happen at arbitrary times. With the above techniques, one would either need as many time intervals $T$ as there are such switches, or several switches would need to be combined into one time bin. In order to avoid these restrictions, this paper introduces the following approach:

- The interface, containing the calls to the network attributes, in particular link speeds, link capacities, and link widths, is made time-dependent, allowing the implementation of arbitrary time-dependent functions behind the interface. This corresponds to the time-aggregated graph technique.

- The implementation presented here uses so-called network change events. The change events are applied to the edges, modifying their attributes (free speed, flow capacity) at arbitrary points in time. A change event is valid until the next change event will be applied.

In this paper we give a description of the simulation framework with a focus on the time dependent aspects. The performance of the simulation framework 
is demonstrated through a case study of the Indonesian city of Padang that faces a high risk of being inundated by an earthquake-triggered tsunami. This work is part of the numerical last-mile tsunami early warning and evacuation information system project ("Last-Mile-Evacuation") [5, 41].

The remainder of the paper is organized as follows. Section 2 gives an overview of the simulation framework with a detailed description of the time dependent aspects. In Section 3, we describe the evacuation scenario that was chosen to demonstrate the simulation framework. Section 4 presents the results of the simulation with respect of the computing performance and the evacuation results. After a short discussion in Section 5, we conclude our work and give some information about future work.

\section{Simulation framework}

The simulation framework is based on the MATSim framework for transport simulation [45]. MATSim is implemented in Java. Since its current implementation is focused on simulation of daily motorized traffic, several adaptations were necessary. The key elements of MATSim are:

- The agent database, where every agent represents one evacuee.

- The simulation network, based on links and nodes.

- The traffic flow simulator, where all the agents' plans are executed.

- The plans generator, which generates an escape plan for every agent.

- There is a mechanism that allows improving the performance of the agents' plans by repeatedly trying to find faster evacuation routes.

\subsection{Synthetic population, plans, agent database}

MATSim always starts with a synthetic population. A synthetic population is a randomly generated population of individuals which is based as much as possible on existing data such as census data. For evacuation, the synthetic population is the collection of all synthetic individuals that are involved in the evacuation.

Every synthetic individual possesses one or several plans. These plans are "intentions" of the synthetic individuals, to be tested in the traffic flow simulation (described later), and scored afterwards. For evacuation, the plans are evacuation strategies. For example, such a strategy may be to leave the building 5 minutes after a second warning, and follow a predetermined route to safety. The collection of agents together with their plans is sometimes called an agent database.

People can have different positions within the city when a warning occurs. For example, they can be at home or at work. Therefore, also in the evacuation context it makes sense to consider MATSim plans in their more conventional meaning, as a description on what a synthetic traveller intends to do during a 
normal day. One can then run a regular traffic flow simulation with these plans, stop it at the time of an evacuation warning, and use the positions of all agents at the time of that warning as the initial condition to the evacuation. This will be the subject of future work.

\subsection{Time-varying network}

The simulation network represents the area that is accessible by the evacuees. In the case of a vehicular evacuation this network consists of all accessible streets. Each street segment defines a link. The parameters of the links are the length, capacity and the free flow speed. For a pedestrian evacuation the links in the simulation network also consist of squares and sidewalks. The flow capacity is given by the width of a link as described in the next section. A good way of creating the simulation network is by extracting the needed information from satellite imagery [66].

For evacuation simulations the network has two kinds of time-varying aspects. First there are the disaster related aspects (i.e. time dependent blocking of links). And second there is the information about (experienced) link travel times, caused by congestion. The time dependent congestion effects are important for the agent replanning (discussed in Sec. 2.5), while the disaster related aspects are also important for the initial plans generation (see Sec. 2.4) and the traffic flow simulator (see Sec. 2.3). Since the object of this work is to develop an evacuation simulation framework for large-scale scenarios, the time-varying aspects should be handled in an efficient way.

\subsubsection{Using arrays for the storage and retrieval of network change events}

The disaster related time-varying aspects are modeled as network change events. A network change event modifies parameters of a link in the network (e.g. free speed or flow capacity). As soon as a link is no longer passable its free speed will be set to zero.

Listing 1 Sample network change event: valid from 03:06 AM, applied to links with id 12487, 12489 and 12491. The change event sets the free speed of the corresponding links to zero.

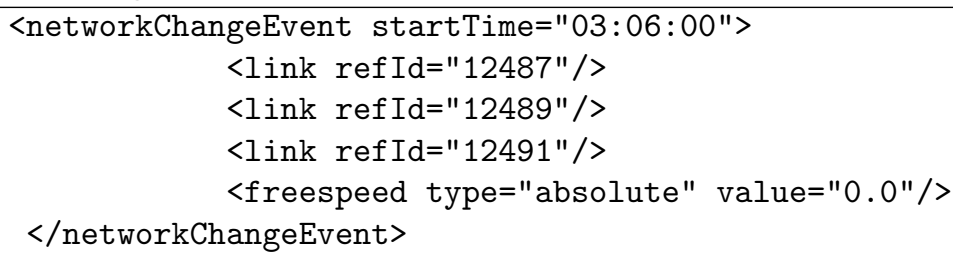

The network change events are stored independent from the network in a separate XML file. Listing 1 shows a sample network change event. The event manipulates the links 12487, 12489 and 12491 at 03:06 AM by setting the free speed to $0 \mathrm{~m} / \mathrm{s}$. The change values are in SI based units. 
The network change events file are loaded at simulation start up and the network change events will be applied to the corresponding links. The attributes of the links that can be influenced by the change events are accessed by time dependent getter methods (e.g. getFreespeed(double time)). These getter methods retrieve the value that is valid for the query time. If there exists a network change event for the given query time, then the value of this network change event will be returned. But, as discussed in Sec. 1, it is not expected that a network change event exists for every link and every time step. If for a given query time no network change event exists, then the value of network change event with the next smaller event time will be returned. The time dependent getter methods give the flexibility to query the attributes in arbitrary chronology.

As a consequence of this flexibility, the retrieval mechanism is not straight forward. Two different approaches have been tested. The first approach that has been tested is to store the network change events in a Java TreeMap. The Java TreeMap implements a red-black tree with a complexity of $O(\log n)$ for get operations. The second approach that has been tested is to store the network change events in an array in chronological order and use a binary search to find the corresponding entry. The complexity for a binary search is also a $O(\log n)$. However, the Java TreeMap cannot operate on primitives (double, int) but the primitives have to be converted into objects (Double, Integer). Since Java 5 this conversion is done automatically (autoboxing). The autoboxing mechanism gives the software developer more flexibility, but produces an overhead that increases the run time. A small benchmark scenario illustrates this issue. For both approaches the retrieval time for network change events was measured. The number of network change events was successively increased. To get a robust result, the time was taken over 10000000 queries. The test was performed on a $2.33 \mathrm{GHz}$ CPU. Fig. 1 shows that the TreeMap implementation is about $20 \%$ slower than the implementation using arrays and binary search. ${ }^{2}$ In consequence, the array approach was chosen to implement the time dependent network.

\subsubsection{Using hash maps for the storage and retrieval of link travel times}

MATSim was originally developed for the simulation of vehicular traffic for large cities or even regions. For this kind of simulation a temporal resolution of 15 min for the link travel times is used: The link travel times are aggregated in 15 min time bins and the travel time values are stored in arrays (one array for each link). For a network consisting of 100000 links, this means a memory consumption of about $75 \mathrm{MB}^{3}$ for the link travel time values.

For a pedestrian evacuation simulation with an expected evacuation time

\footnotetext{
${ }^{2}$ In fact, we and others [47] (Item 23) consistently find that in situations with few or no insertions or removals after initialization, the array-based approach is faster.

${ }^{3}$ For $24 h$ day there are $4 * 24$ time bins, each time bin holds one double value (64 bit) and there are 100000 links $(4 * 24 * 100000 * 64$ bit $\approx 75 \mathrm{MB})$.
} 


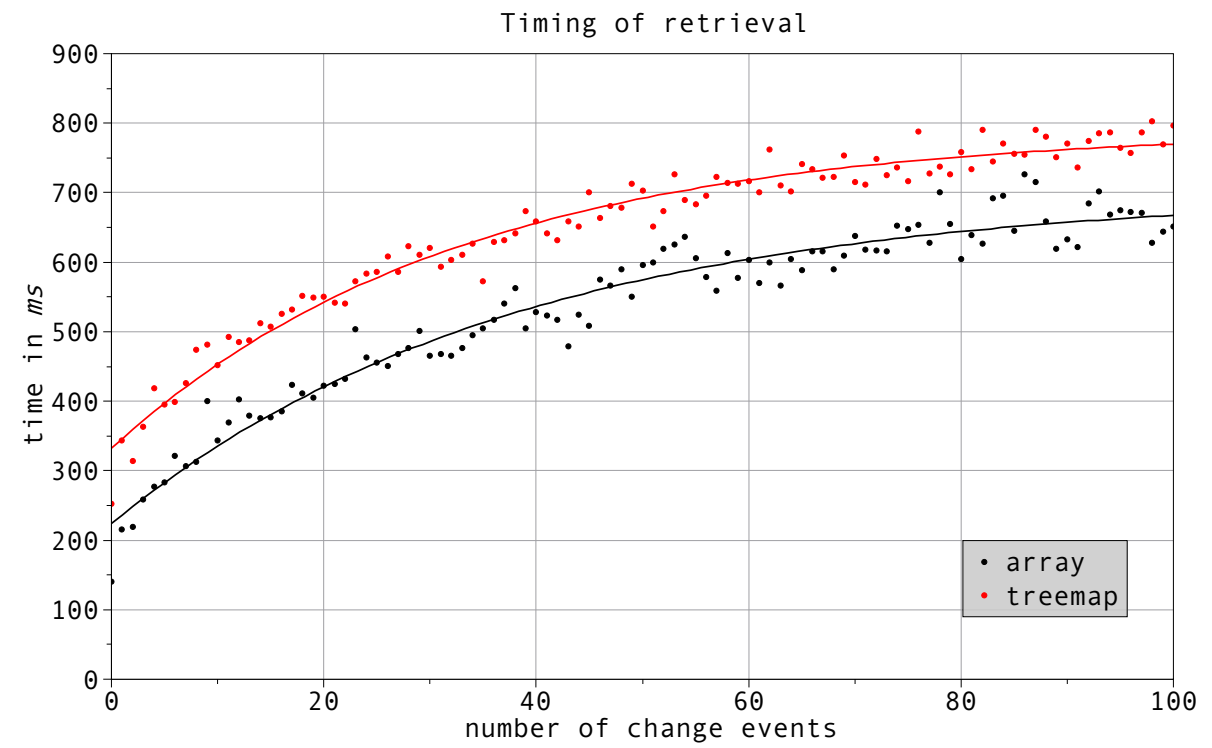

Figure 1: Comparison of the runtime performance for two different implementations of the network change event retrieval. The array based approach using binary search is about $20 \%$ faster then the approach using a Java TreeMap. The complexity of both approaches is $O(\log n)$.

of one or two hours, a resolution of $15 \mathrm{~min}$ is too coarse. However, a finer resolution increases the amount of memory that is needed. To overcome this problem, the array based implementation was replaced by an implementation using Java HashMap. There is a HashMap for each link that stores the link travel times. But only for those travel time bins where at least one agent has entered the link, a travel time value is stored. This means that if for a given travel time bin no agent enters the corresponding link, then nothing is stored (and the free speed travel time will be used). Depending on the scenario this can save a lot of memory.

An analytic appraisal of the memory usage or the needed execution time in Java is difficult and depends, besides others, on the virtual machine and its garbage collection mechanism, and on the specific scenario. Therefore it was decided to compare the HashMap based approach with the array based approach through a benchmark scenario. In the benchmark scenario, all link travel times of the simulation described in Sec. 3 have been recorded and aggregated for different travel time bin sizes. Beginning with a travel time bin size of $15 \mathrm{~min}$ and ending with a travel time bin size of $1 \mathrm{~min}$, the memory consumption, the time for storing and aggregating and the time for the retrieval of the link travel times have been measured.

The results of this benchmark test are shown in Fig. 2. At the top there is a diagram comparing the memory usage of both approaches. Clearly, the HashMap based approach consumes considerably less memory than the array 


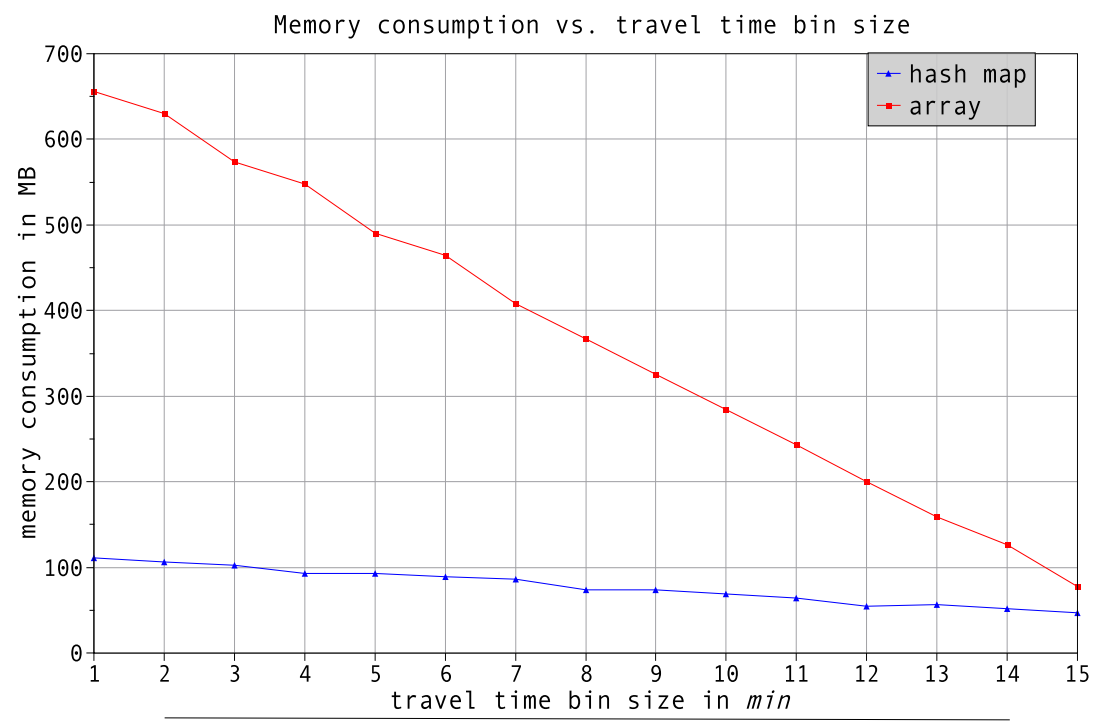

Run time vs. travel time bin size

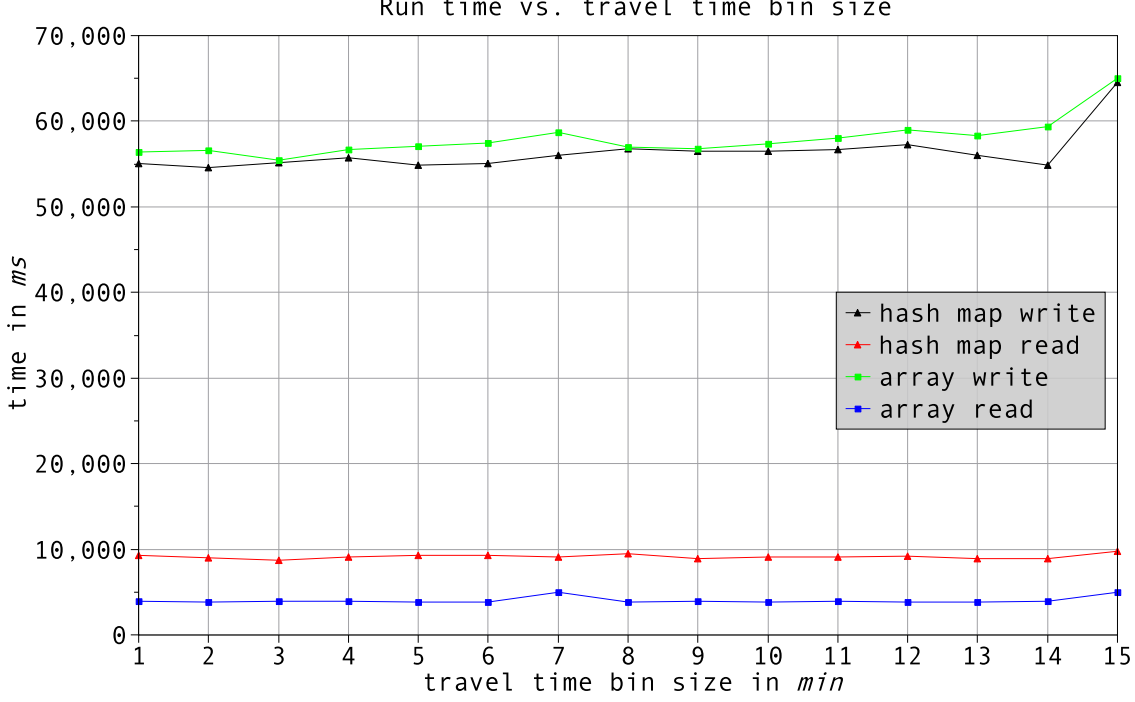

Figure 2: Comparison of two different implementations of the storage and retrieval mechanism for the time dependent link travel times. Top: comparison of the memory consumption. The Java HashMap based approach consumes considerable less memory than an array based implementation. Bottom: comparison of the runtime performance. For write operations both approaches have almost equal execution times. The array based approach is faster for read operations. 
based implementation. In particular, at 1-min time resolution the array-based implementation consumes about $650 \mathrm{MB}$ of memory, which, together with the memory requirements of the remainder of the package, would make simulations on today's ordinary desktop computers impossible. The diagram at the bottom compares the runtime for storage and aggregation (write) and for retrieval of the link travel times (read). The time needed for write operations is almost equal for both approaches. For read operations the array approach is faster. At the same time, the execution time for a read operation is much smaller than for a write operation. The underlying simulation run simulates the evacuation of about 320000 agents. On average each agent has to traverse 23.5 links before she reaches the safe area. This means there are approximately 75000000 different link travel times that have to be aggregated and stored. For the benchmark scenario there were approximately 250000000 synthetic read operations ${ }^{4}$. Overall, for the time dependent link travel times retrieval, the small disadvantage of the hash map approach with respect to the runtime can be tolerated for the sake of much lower memory consumption.

\subsection{Traffic flow simulator}

The traffic flow simulation is implemented as a queue simulation, where each street (link) is represented as a FIFO (first-in first-out) queue with three restrictions [18]. First, each agent has to remain for a certain time on the link, corresponding to the free speed travel time. Second, a link flow capacity is defined which limits the outflow from the link. If, in any given time step, that capacity is used up, no more agents can leave the link. Finally, a link storage capacity is defined which limits the number of agents on the link. If it is filled up, no more agents can enter this link. The difference to standard queueing theory is that agents (particles) are not dropped but spill back, causing congestion. An illustration of the queue model is shown in Fig. 3 a). The parameters of the model are:

- Link minimum width $w$

- Link area $A$

- Link length $l$

- Flow capacity $F C=w * C_{\max }=w * 1.3 \frac{p}{m * s}$

- Free flow speed $v_{\max }=1.66 \frac{\mathrm{m}}{\mathrm{s}}$

- Storage capacity $S C=A * D_{\max }=A * 5.4 \frac{p}{m^{2}}$

where $C_{\max }$ is the maximum flow capacity per unit width, and $D_{\max }$ is the maximum density per unit area. The flow capacity of 1.3 persons/second per

\footnotetext{
${ }^{4}$ The network for the scenario consists of 16978 links. For each link the travel time was queried 20 times for 720 different time steps. $(16978 * 20 * 720 \approx 250000000)$
} 

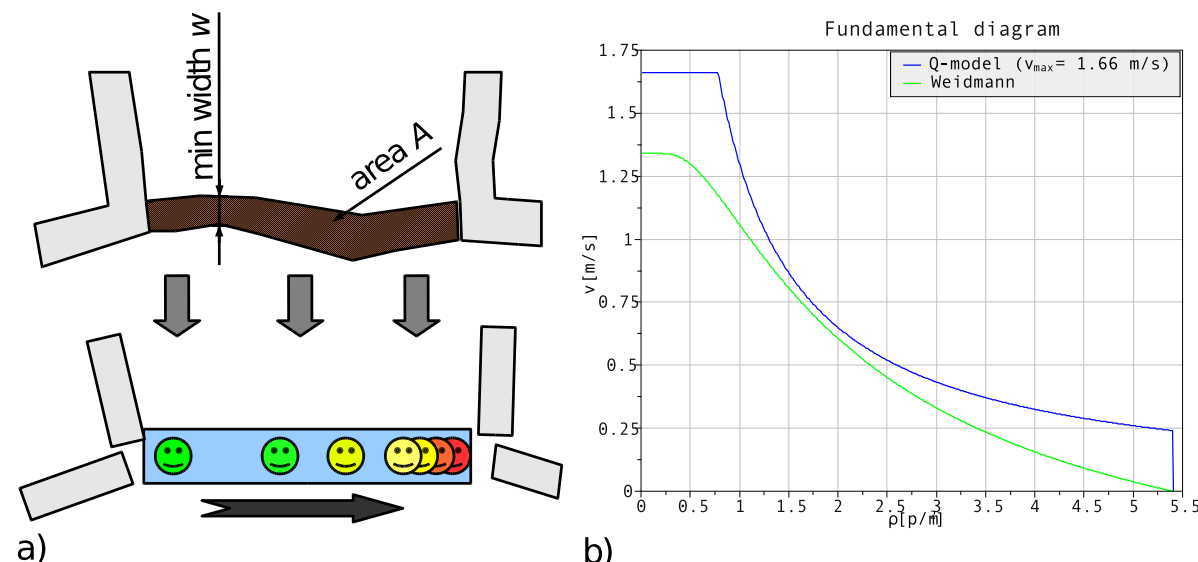

b)

Figure 3: Functioning of the queue model is shown in (a) and its corresponding fundamental diagram in (b).

meter of cross section is a fairly robust empirical parameter [68, 13], and it is the most important parameter to determine bottlenecks. The remaining parameters have been chosen to approximate Weidmann's fundamental diagram [68]. For more details, see [40].

\subsection{Plans generation}

Initial plans use the shortest path (according to free speed travel time) out of the evacuation area for all agents. Within the MATSim framework a shortest path router based on Dijkstra's shortest path algorithm [12] has been implemented. This router finds the shortest path in a weighted graph from one node to any other, whereby the actual weights for a link are defined by a timedependent cost function. Since the city has to be evacuated as fast as possible, the weights represent the (expected) travel time.

There is, however, no particular node as the target of the shortest path calculation, as the evacuees have more than one safe place to run to. Instead, in the underlying domain every node outside the evacuation area is a possible destination for an agent that is looking for an escape route. To resolve this, the standard approach (e.g. [44]) is to extend the network in the following way: All links which lead out of the evacuation area are connected, using virtual links with infinite flow capacity and zero length, to a special "evacuation node". Doing so, Dijkstra's algorithm will always find the shortest route from any node inside the evacuation area to this evacuation node.

\subsection{Agent learning}

During the simulation, each evacuee optimizes his/her personal evacuation route to find the fastest escape route. At this point two different routing solutions are considered: (1) A "shortest path" routing solution, where every evacuee follows the path that would be fastest in an empty network. (2) A "Nash 
equilibrium" approach, where, via iterations every evacuating person attempts to find a route that is optimal for him-/herself under the given circumstances including congestion. Both approaches can be considered as benchmarks: the first as one where congestion effects are not taken into account in the path choice; the second one as one which might be achieved by appropriate training or guidance while maintaining acceptability in the sense that no person could gain by deviating from this solution. This will be discussed in more detail in Sec. 5.

At the end of every iteration, every agent will score the performed plan. The score of a plan is the negative of its execution time (i.e. of the necessary time to evacuate). The scored plans remain in the agents' memory for further iterations. Two different learning strategies have been applied for the learning procedure.

- The "ReRoute" strategy generates new plans with new evacuation routes based on the information of the experienced travel times from the last run. This uses the router described in the previous section, but now using time-variant link travel times (Sec. 2.2.2) as link costs. The link travel times are aggregated into 3 min time bins.

- The other strategy, "ChangeExpBeta", decides if the just performed plan should be used again, or if a random plan out of the memory should be selected for the next iteration. The probability to change the selected plan is calculated as:

$$
p_{\text {change }}=\min \left(1, \alpha * e^{\beta *\left(s_{\text {other }}-s_{\text {current }}\right) / 2}\right),
$$

with:

$-\alpha$ : The probability to change if both plans have the same score

- $\beta$ : A sensitivity parameter

$-s_{\{\text {other,current }\}}:$ The score of the other/current plan

Eq. 1 describes a process which slowly converges towards the logit model probability distribution

$$
p_{j}=\frac{e^{\beta * s_{j}}}{\sum_{i} e^{\beta * s_{i}}},
$$

where $p_{i}$ is the probability for plan $i$ to be selected and $s_{i}$ its current score. This makes convergence to a steady state smoother than when all choices are made in every iteration.

A strategy selector decides for every agent which of the strategies (ReRoute or ChangeExpBeta) will be used. Each strategy is selected with a certain probability. These probabilities are assigned before the simulation starts, but they can be varied during the iterations.

After re-planning every agent has a selected plan that will be executed in the next iteration. Repeating this iteration cycle of learning, the agents' behavior 
will move towards a Nash equilibrium. If the system were deterministic, then a state where every agent uses a fixed plan that is always a best response to the last iteration would be a fixed point of the iterative dynamics, and at the same time a Nash Equilibrium since no agent would have an incentive to unilaterally deviate. Since, however, the system is stochastic, this statement does not hold, and instead we look heuristically at projections of the system, e.g. the average evacuation time. From experience it is enough to run 100 iterations until the iterative dynamics has reached a steady state. In most (but not all) evacuation situations, the Nash equilibrium leads to a shorter overall evacuation time than when everybody moves to the geographically nearest evacuation point.

\section{Scenario}

The aim of this work is to find feasible solutions for the evacuation of the city of Padang in the case of a tsunami. There are several aspects that have to be taken into consideration. At first one needs a synthetic population for the city. In this case study it is assumed that all people are at home. The information about the distribution of the population was derived from existing census data [7]. Approximately 320000 people are living in the endangered area. This means that 320000 agents have been created for the simulation.

As discussed in Sec. 2.3, the traffic flow simulation is performed on a network representing the walkable area of the city. The street map was extracted from satellite imagery by remote sensing technologies [65] and converted into a graph [41]. The resulting simulation network consists of 6289 nodes and 16978 unidirectional links.

Another important aspect is the information about safe places. In the future it is planned to identify buildings that are suitable for a vertical evacuation. For the time being we use a simpler approach: All areas with an elevation of more the $10 \mathrm{~m}$ above sea level are defined as safe. Fig. 4 shows an image of the city with the safe area. However, based on models of small-scale flooding and inundation dynamics of the tsunami [22] it is not expected that all the area below $10 \mathrm{~m}$ will be flooded. Based on these simulations, one also learns that the estimated time between the earthquake and the inundation of the city is about $28 \mathrm{~min}$. The results are backed by the results of large-scale tsunami simulations for the west coast of Sumatra Island [46]. Adding this to the simulation, the agents were made to learn a more risk averse behavior: they are not only trying to reach the safe area as fast as possible, but they also try to increase the distance to the endangered areas. In some places the flooding will reach locations that are more than $2 \mathrm{~km}$ away from the shoreline.

The inundation data was provided by [22] as a time series of flooding heights for $(x, y)$-coordinates with a temporal resolution of $1 \mathrm{~min}$ and a spatial resolution of $3 \mathrm{~m}$. The queue model reproduces the inundation as a time dependent network by varying the free speed parameter of the links. The free speed, as discussed in Sec. 2.2, for the not inundated link is $1.66 \mathrm{~m} / \mathrm{s}$ and the free speed for an inundated link is $0 \mathrm{~m} / \mathrm{s}$. This means as soon as a link is flooded its free speed will be set to $0 \mathrm{~m} / \mathrm{s}$. The router will, in consequence, try avoid the link 


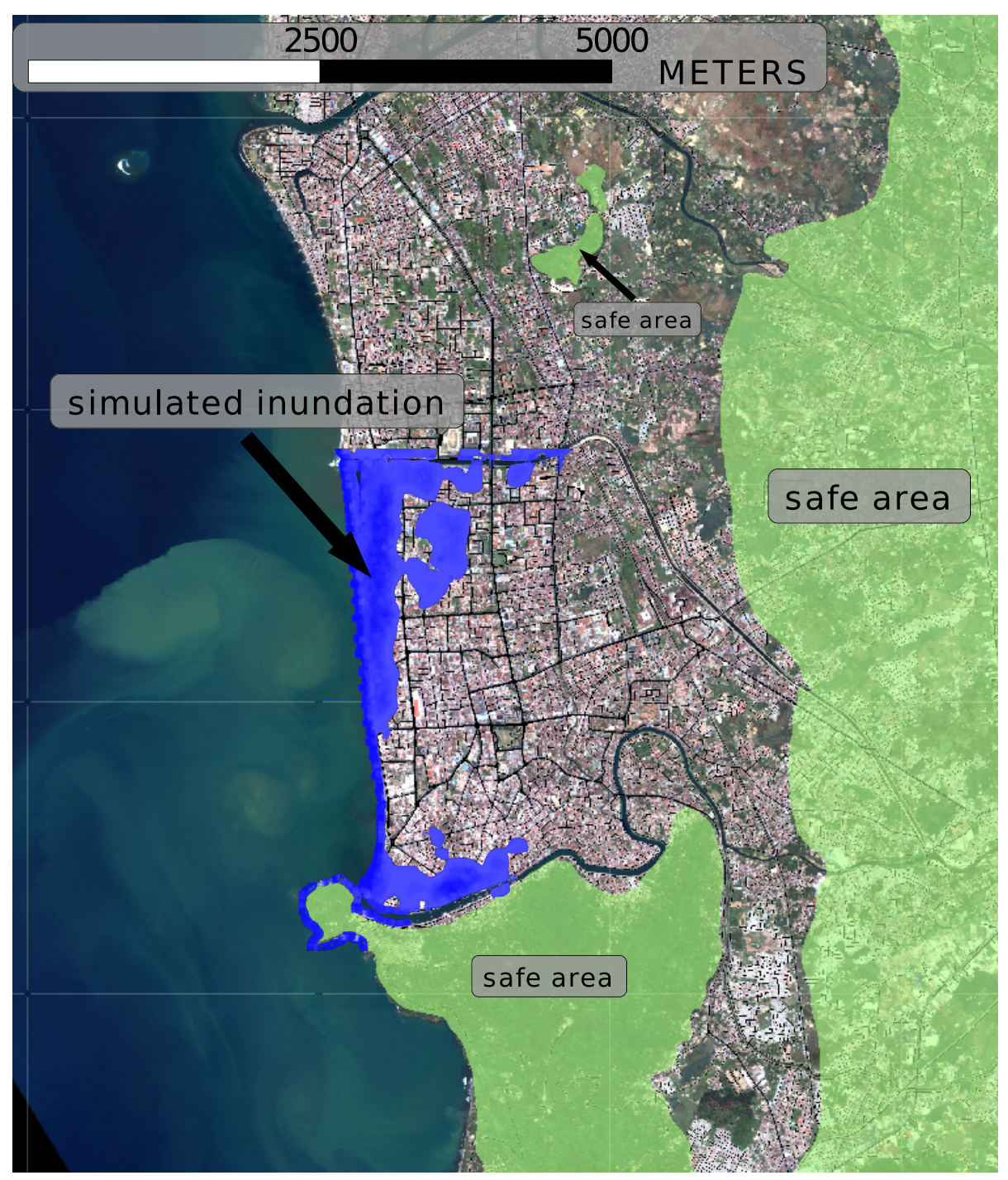

Figure 4: Satellite imagery of the city shows the safe area (light green) and some preliminary results of the flooding simulation (blue area). Satellite imagery by the German Aerospace Center, Oberpaffenhofen (2007) 
at these times. And if, nevertheless, an agent happens to be on this link at such a time, it will remain stuck there forever.

As explained above, we applied two different strategies for learning to the simulation. The ReRoute strategy finds a new evacuation route for an agent, based on the experienced travel times of the former iteration. The ChangeExpBeta strategy implements a discrete-choice model that assigns a plan from the agent's memory with a probability depending on the score of the plan. In the current setup the probability of being chosen for the ReRoute strategy is $10 \%$ and $90 \%$ for ChangeExpBeta. This setup gives a fair arrangement between exploration and exploitation. If the probability for ReRoute (i.e. exploration) is too low, then it could be that some promising routes will never be discovered. On the other hand, if the probability for ReRoute is very high, the system tends to fluctuate and will not convert to a steady state. The system would change so fast that the agents would not get a chance to exploit their knowledge about the system.

\section{Results}

Three different simulation runs were conducted. Run 1 corresponds to the previous version of the evacuation simulation, without the time-dependent network. Run 2 now includes the time-dependent network, which means that the simulation now includes the effects of links that get flooded during the evacuation. Run 3 is similar to run 1 except that here a so called $10 \%$ scenario was chosen. This means only a $10 \%$ sample of the agents have been loaded onto the network. To get correct results the flow capacity and storage capacity of all links was also scaled by a factor of 0.1. Run 3 was conducted to see how the runtime performance scales with the size of the scenario.

All runs were performed on computer with a Dual-Core CPU at $3 \mathrm{GHz}$ running a 64-Bit Java version on a Linux operation system. The simulation runs were stopped after 200 iterations of learning. Table 1 compares the runtime performance and memory consumption of the three runs. It is shown that

\begin{tabular}{|l|r|r|r|}
\hline run & overall runtime & avg. runtime per iteration & max. memory usage \\
\hline \hline 1 & $370 \mathrm{~min}$ & $111 \mathrm{~s}$ & $3856 \mathrm{MB}$ \\
\hline 2 & $400 \mathrm{~min}$ & $120 \mathrm{~s}$ & $3809 \mathrm{MB}$ \\
\hline 3 & $77 \mathrm{~min}$ & $23 \mathrm{~s}$ & $1974 \mathrm{MB}$ \\
\hline
\end{tabular}

Table 1: Runtime performance and memory consumption of the three different runs.

runtime performance of run 2 (with time dependent network) is slightly worse compared to run 1 (without time dependent network). Both runs need a similar amount of memory. This results show that applying time dependent networks virtually make no difference regarding the runtime performance and memory consumption. Thus the time dependent functionality has been implemented in an efficient way. 


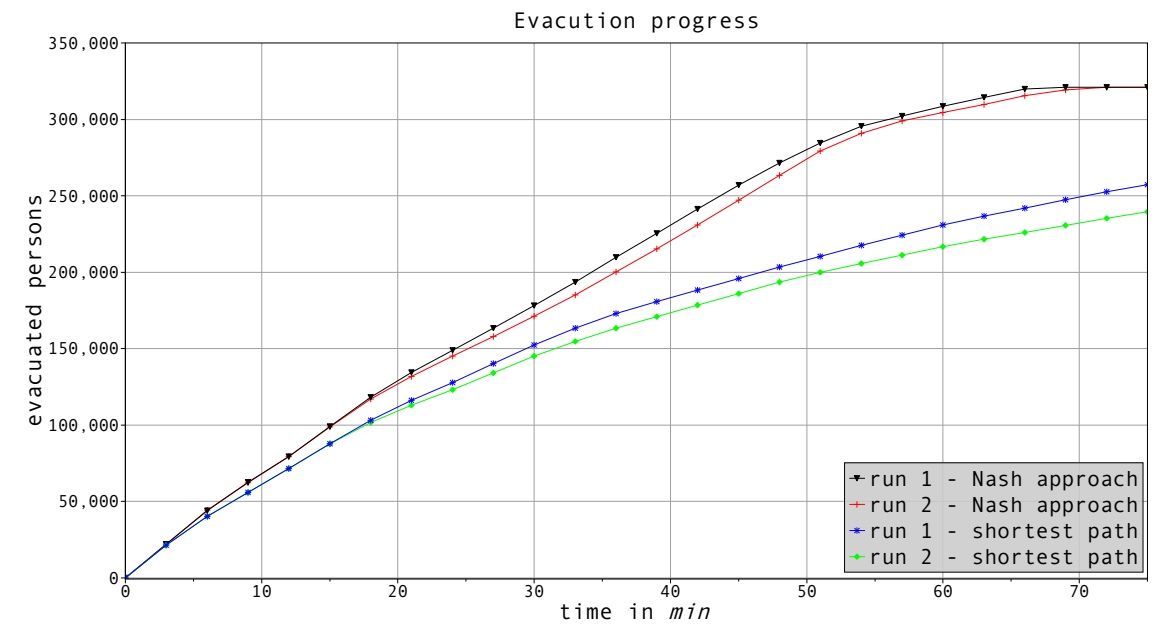

Figure 5: Comparison of the evacuation curves of the first iteration ("shortest path" solution) and the last iteration ("Nash equilibrium" approach) of run-new and run-old. The curves are truncated at $75 \mathrm{~min}$.

Compared to the first and the second run run 3 is about 5 times faster and consumes only the half amount of memory. Since "10\%-runs" still yield useful results, this means one could also run much larger scenarios on the current hardware.

It is common to show the results of an evacuation simulation through evacuation curves. Fig. 5 shows such curves for the first and the last iteration of run 1 and run 2. These curves show that the evacuation without time dependent network is slightly faster in both the first and the last iteration. In the last iteration the evacuation takes about 65 min without time dependent network (run 1) compared to 75 min with time dependent network (run 2). In run 2 the agents are aware of the inundation; in run 1, they are not. It makes sense that in run 2 the evacuation takes longer, since the agents take an additional constraint into account.

Fig. 6 shows a GIS analysis of the network clearance time on a $350 \mathrm{~m}$ grid. The colors of the squares indicate the difference of the clearance time for run 1 vs. run 2. A green color indicates that the clearance was faster in run 2 (with time dependent network) and a red color indicates that it was slower. It is shown that in run 2 the clearance of the coastal area is much faster than in run 1. Especially the southern parts of the city are evacuated more then $10 \mathrm{~min}$ earlier. This is a major progress in finding feasible solutions for the evacuation of Padang.

Fig. 7 shows how different solutions ("shortest path" solution from run 1, "Nash" solution from run 1, "Nash" solution from run 2) perform when executed with the time-dependent network and the incoming tsunami wave. In particular, highly endangered agents are colored in red. It is clearly visible that 


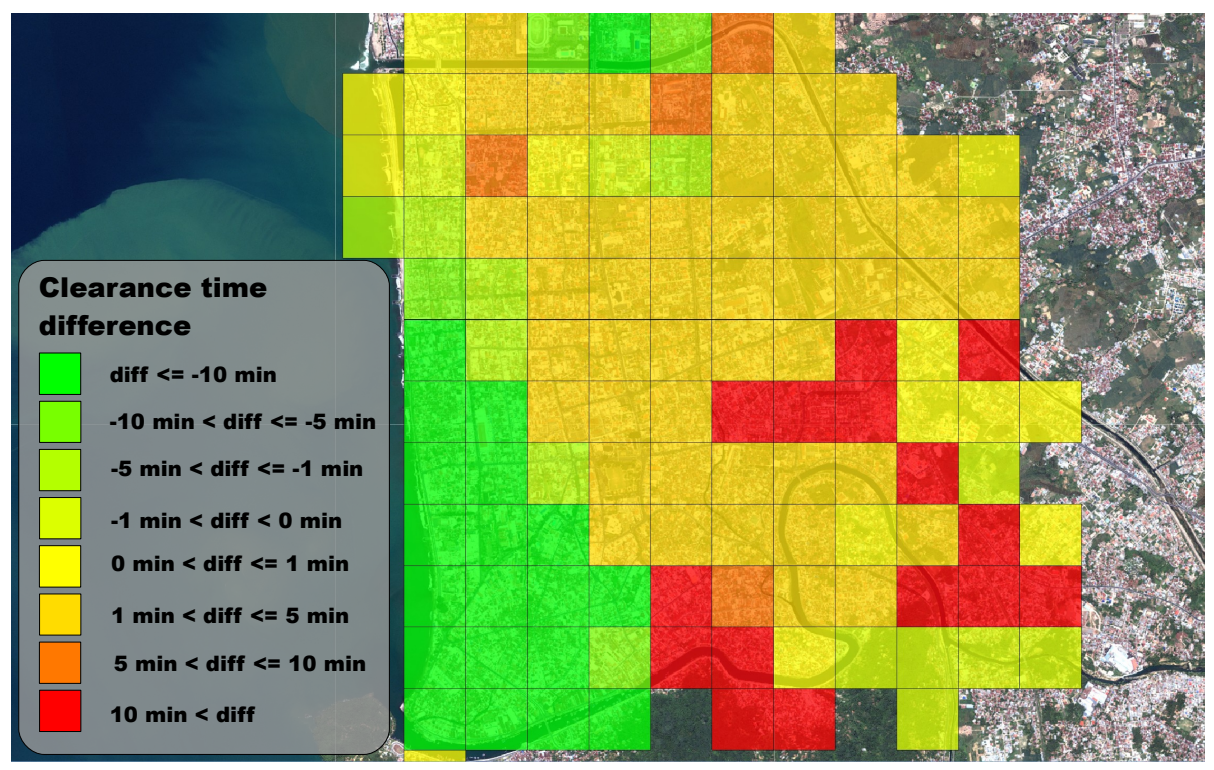

Figure 6: This figure shows a GIS analysis of the network clearance time on a $350 \mathrm{~m}$ grid. The color of the squares indicate the difference of the clearance time for the simulation run without time dependent network compared to the simulation run where the time dependent network approach has been applied. A green color indicates that the clearance was faster with time dependent network and a red color indicates a faster clearance without time dependent network. The actual values are given in the legend of the figure (e.g. a green color means that the clearance time with time dependent network was at least 10 min faster than without). It is shown, that with time dependent network the clearance time for the highly endangered coastal area decreases on the cost of increase of clearance time in the hinterland.

the "shortest path" solution does not leave enough time to evacuate the costal strip. Furthermore, the "Nash" solution of run 1 would also not leave enough time to evacuate the highly endangered area within $30 \mathrm{~min}$. The results from the "Nash equilibrium" approach of run 2 seem to be more feasible.

\section{Discussion}

The simulations concentrate on two types of agent behaviors: One where every agent follows the shortest path to the safe area; one where a Nash equilibrium is reached. Both can be considered as benchmarks:

- The first as one where agents are rational about their path choice, but unaware of congestion effects.

- The second as a solution that could be reached by training, assuming that agents follow the training solution also in the real situation.

In panic situations, people tend to be irrational and to display herd behavior [28]. Still, if even the Nash equilibrium solution does not leave enough time, 


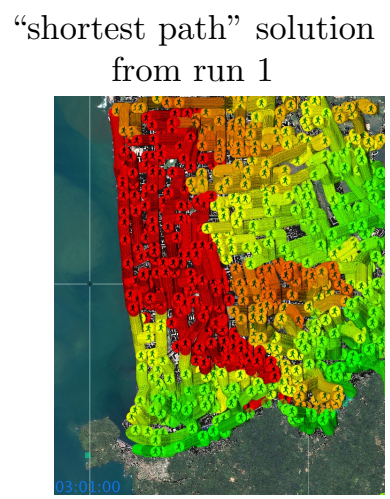

\section{"Nash" solution from run 1}
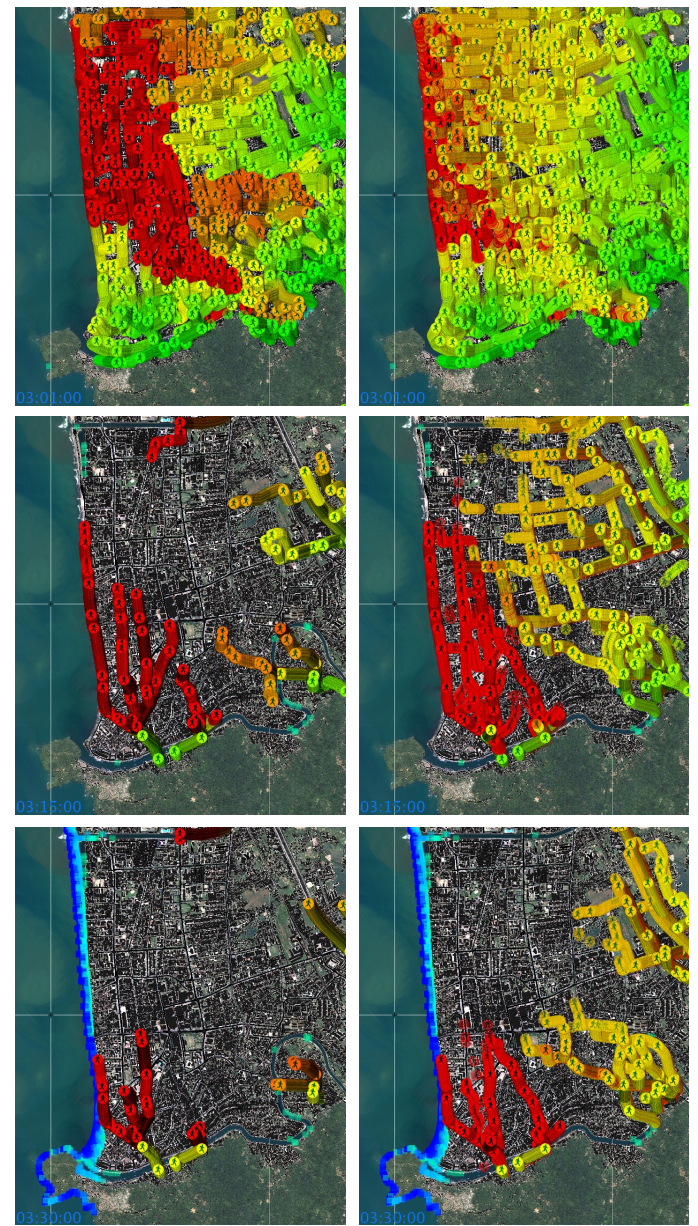
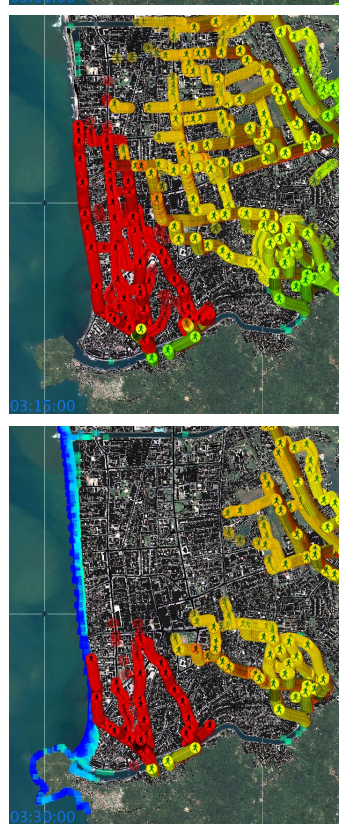

"Nash" solution from run 2
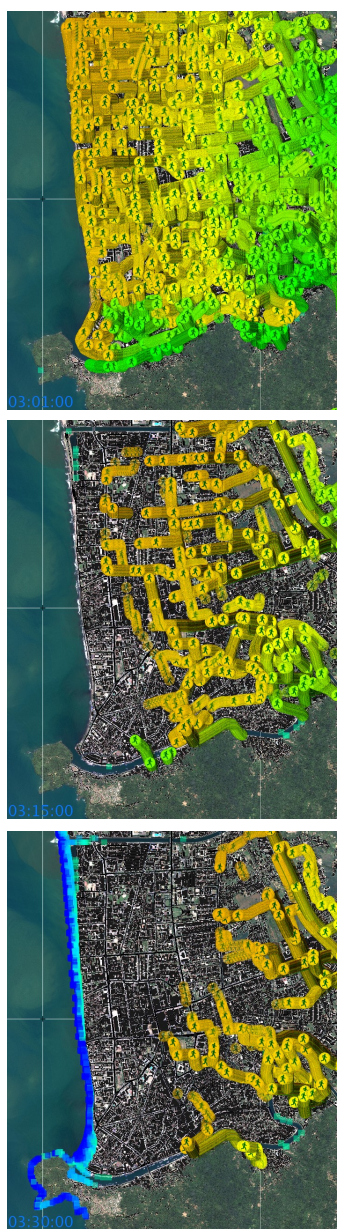

Figure 7: Visualizer snapshots of the evacuation progress. The evacuation starts at 03:00 AM and the snapshots are taken after $1 \mathrm{~min}, 15 \mathrm{~min}$ and $30 \mathrm{~min}$. (left) The three snapshots in the left column show the evacuation progress for the "shortest path" solution of run 1 performed on the time dependent network. (center) The snapshots in the center column show the "Nash equilibrium" approach of run 1 also performed on a time dependent network. (right) The snapshots in the right column show the result of the "Nash equilibrium" approach of run 2. The agents are colorized with respect to the time they need to evacuate. The evacuation time increases as the color moves from green to yellow to red. Agents that did not manage to evacuate the area that will be inundated are also colored red. Note in particular some highly endangered agents in the shortest path solution due to congestion. The "Nash" solution of run 1 is even worse. 
then this would be a strong indicator that major measures would need to be taken to rectify the situation.

It should also be stated that Nash equilibrium and system optimum do not need to coincide - i.e. that solutions even better than the Nash equilibrium might be possible. Such solutions would, however, be unstable in the sense that people would have an incentive to deviate. Such solutions seem even more improbable than Nash equilibrium solutions.

Finally, one should mention that MATSim already contains the first hooks towards en-route replanning [31]. This would allow to add situation-based behavior into the simulation.

Another issue concerns the mode choice: The investigation assumes that all evacuation is done by foot while it might be reasonable to assume that some people use cars or cycles, and they might even leave vehicles in the street to continue on foot if progress by vehicle becomes too slow. For the time being, such issues are not considered. The queue model could, to a certain extent, be parameterized to deal with mixed traffic, as long as all modes move with the same speed. Beyond that, one would arguably need to switch to a true two-dimensional model such as [28] or [35]. Such models could still operate on networks [20].

In the current base case it is assumed that all people are at home. Currently we are working on more detailed picture of the population. Based on census data and the results of a survey with 1000 households, that took place in April/Mai 2008 [5], we are developing a synthetic population with individual daily plans. From this synthetic population it will be possible to derive a model of the population distribution at any time of day. In future work it is also planned to integrate tsunami proof shelters into the simulation framework. Therefore the simulation framework could be extended in a way to find optimal locations for the tsunami proof shelters.

\section{Conclusions}

We introduced a microscopic pedestrian simulation framework for large-scale evacuations. It is implemented as a Multi Agent Simulation, where every agent tries to optimize its individual evacuation plan in an iterative way. The flooding information is modeled as network change events in the simulation framework, which, when such events are comparatively rare, is a much sparser representation than time-expanded or time-aggregated graphs. The network change events approach could be easily adapted to other scenarios, for example for modeling accidents.

The simulation framework is demonstrated through a case study based on a tsunami evacuation of the Indonesian city of Padang. The simulation gives plausible results regarding the predicted evacuation time and process. The runtime performance shows that this approach is well suited for large scale scenarios. With state of the art hardware it is no problem to simulate much larger scenarios with over one million agents. 


\section{Acknowledgments}

This project was funded in part by the German Ministry for Education and Research (BMBF), under grants numbers 03G0666E ("last mile") and 03NAPI4 ("Advest"). We would like to thank Hubert Klüpfel for the fruitful discussion about pedestrian evacuation simulations.

\section{References}

[1] Pedestrian and Evacuation Dynamics. Proceedings of the 4th international conference, Wuppertal, 2008. Springer, Berlin, 2008.

[2] R. Alsnih and P. Stopher. A review of the procedures associated with devising emergency evacuation plans. Working Paper ITS-WP-04-04, Institute of Transport Studies, The University of Sydney and Monash University, 2004. See www.itls.usyd.edu.au/publications/working_papers/wp2004/its_wp_0404.pdf.

[3] B. Barrett, B. Ran, and R. Pillai. Developing a dynamic traffic management modeling framework for hurricane evacuation. Paper 00-1595, Transportation Research Board Annual Meeting, Washington, D.C., 2000.

[4] M. Bierlaire, G. Antonini, and M. Weber. Behavioral dynamics for pedestrians. In K. Axhausen, editor, Moving through nets: The physical and social dimensions of travel. Elsevier, 2003.

[5] J. Birkmann, S. Dech, N. Goseberg, H. Klüpfel, G. Lämmel, F. Moder, K. Nagel, M. Oczipka, T. Schlurmann, N. Setiadi, F. Siegert, G. Strunz, and H. Taubenböck. Numerical last-mile tsunami early warning and evacation information system ("Last-Mile - Evacuation"). In GEOTECHNOLOGIEN Science Report No. 10: "Early Warning Systems in Earth Management", Osnabrück, October 2008.

[6] J. Birkmann, S. Dech, G. Hirzinger, R. Klein, H. Klüpfel, F. Lehmann, C. Mott, K. Nagel, T. Schlurmann, N. Setiadi, F. Siegert, and G. Strunz. Numerical last-mile tsunami early warning and evacuation information system. In L. Stroink, editor, GEOTECHNOLOGIEN Science Report No. 10: "Early Warning Systems in Earth Management", Technical University Karlsruhe, October 2007. Die Deutsche Biliothek.

[7] BPS. Kecamatan Dalam Angka - Subdistricts in Numbers. Satistical bureau (BPS) Kota Padang, Padang, 2005.

[8] E. Cascetta and C. Cantarella. A day-to-day and within-day dynamic stochastic assignment model. Transportation Research A, 25A(5):277-291, 1991. 
[9] N. Cetin, A. Burri, and K. Nagel. A large-scale agent-based traffic microsimulation based on queue model. In Proceedings of Swiss Transport Research Conference (STRC) Monte Verita, CH, 2003. See www.strc.ch. Earlier version, with inferior performance values: Transportation Research Board Annual Meeting 2003 paper number 03-4272.

[10] X. Chen and F. Zhan. Agent-based modeling and simulation of urban evacuation: Relative effectiveness of simultaneous and staged evacuation strategies. Paper 04-0329, Transportation Research Board Annual Meeting, Washington, D.C., 2004.

[11] Y.-C. Chiu, P. Korada, and P. Mirchandani. Dynamic traffic management for evacuation. Paper 05-2369, Transportation Research Board Annual Meeting, Washington, D.C., 2005.

[12] E. Dijkstra. A note on two problems in connexion with graphs. Numerische Mathematik, 1:269 - 271, 1959.

[13] P. DiNenno, editor. SFPE Handbook of Fire Protection Engineering. National Fire Protection Association, Boston, 2nd edition, 1995.

[14] I. Farkas. pedsim source code, accessed 2008. See pedsim.elte.hu.

[15] E. Galea. Simulating evacuation and circulation in planes, trains, buildings and ships using the EXODUS software. In Schreckenberg and Sharma [60], pages 203-225.

[16] E. R. Galea, editor. Pedestrian and Evacuation Dynamics. Proceedings of the 2nd international conference, London, 2003. CMS Press, University of Greenwich, UK, 2003.

[17] P. Gattermann, N. Waldau, and M. Schreckenberg, editors. Pedestrian and Evacuation Dynamics. Proceedings of the 3rd international conference, Vienna. Springer, Berlin, 2006.

[18] C. Gawron. An iterative algorithm to determine the dynamic user equilibrium in a traffic simulation model. International Journal of Modern Physics C, 9(3):393-407, 1998.

[19] B. George, S. Kim, and S. Shekhar. Spatio-temporal network databases and routing algotihms: A summary of results. In Proceedings of International Symposium on Spatial and Temporal Databases (SSTD`07), 2007.

[20] C. Gloor, P. Stucki, and K. Nagel. Hybrid techniques for pedestrian simulations. In Cellular automata, Proceedings, number 3305 in Lecture Notes in Computer Science, pages 581-590. Springer, 2004.

[21] C. Gloor, P. Stucki, and K. Nagel. Hybrid techniques for pedestrian simulations. In Proceedings of Swiss Transport Research Conference (STRC) Monte Verita, CH, 2004. See www.strc.ch. 
[22] N. Goseberg, A. Stahlmann, S. Schimmels, and T. Schlurmann. Highlyresolved numerical modeling of tsunami run-up and inundation scenarios in the city of Padang, West Sumatra. In Interantional Conference on Coastal Engineering, Hamburg, in press.

[23] S. Hamacher, H.W. Tjandra. Mathematical modelling of evacuation problems: A state of art. Berichte des Fraunhofer ITWM, 24:1-38, 2001.

[24] A. Han. TEVACS: Decision support system for evacuation planning in Taiwan. Journal of Transportation Engineering, 116(6):821-830, 1990.

[25] L. Han and F. Yuan. Evacuation modeling and operations using dynamic traffic assignment and most desirable destination approaches. Paper 052401, Transportation Research Board Annual Meeting, Washington, D.C., 2005 .

[26] D. Helbing, L. Buzna, A. Johansson, and T. Werner. Self-organized pedestrian crowd dynamics: experiments, simulations and design solutions. Transportation Science, 39:1-24, 2005.

[27] D. Helbing, I. Farkas, P. Molnar, and T. Vicsek. Simulation of pedestrian crowds in normal and evacuation situations. In Schreckenberg and Sharma [60], pages 21-58.

[28] D. Helbing, I. Farkas, and T. Vicsek. Simulating dynamical features of escape panic. Nature, 407:487-490, 2000.

[29] A. Hobeika and C. Kim. Comparison of traffic assignments in evacuation modeling. IEEE Transactions on Engineering Management, 45(2):192-198, 1998.

[30] S. Hoogendoorn, P. Bovy, and W. Daamen. Microscopic pedestrian wayfinding and dynamic modelling. In Schreckenberg and Sharma [60], pages $123-154$.

[31] J. Illenberger, G. Flötteröd, and K. Nagel. Enhancing MATSim with capabilities of within-day re-planning. In Proceedings of the 2007 IEEE Intelligent Transportation Systems Conference, pages 94-99, Seattle, WA, Sep 30 - Oct 32007.

[32] M. Jafari, I. Bakhadyrov, and A. Maher. Technological advances in evacuation planning and emergency management: Current state of the art. Final Research Reports EVAC-RU4474, Center for Advanced Infrastructure and Transportation (CAIT), Rutgers University, NJ, 2003.

[33] M. Jha, K. Moore, and B. Pashaie. Emergency evacuation planning with microscopic traffic simulation. Paper 04-2414, Transportation Research Board Annual Meeting, Washington, D.C., 2004. 
[34] D. Kaufman and R. Smith. Fastest paths in time-dependent networks for intelligent vehicle-highway systems application. Journal of Intelligent Transportation Systems, 1:1-11, 1993.

[35] H. Klüpfel. The simulation of crowd dynamics at very large events - Calibration, empirical data, and validation. In Gattermann et al. [17].

[36] H. Klüpfel, T. Meyer-König, A. Keßel, and M. Schreckenberg. Simulating evacuation processes and comparison to empirical results. In M. Fukui et al, editor, Traffic and granular flow '01, pages 449-454. Springer, Berlin Heidelberg New York, 2003.

[37] E. Kohler, K. Langtau, and M. Skutella. Time-expanded graphs for flowdependent transit times. In 10th Annual European Symposium on Algorithms, pages 599-611, 2002.

[38] E. Kuligowski. Review of 28 egress models. Technical report, National Institute of Standards and Technology (NIST), Gaithersburg, MD, 2004.

[39] E. Kwon and S. Pitt. Evaluation of emergency evacuation strategies for downtown event traffic using a dynamic network model. Paper 05-2164, Transportation Research Board Annual Meeting, Washington, D.C., 2005.

[40] G. Lämmel, M. Rieser, and K. Nagel. Bottlenecks and congestion in evacuation scenarios: A microscopic evacuation simulation for large-scale disasters. In 5th Workshop on Agents in Traffic and Transportation (ATT) @ Autonomous Agents and Multiagent Systems (AAMAS) '08, Estoril, Portugal, May 2008.

[41] G. Lämmel, M. Rieser, K. Nagel, H. Taubenböck, G. Strunz, N. Goseberg, T. Schlurmann, H. Klüpfel, N. Setiadi, and J. Birkmann. Emergency preparedness in the case of a tsunami - evacuation analysis and traffic optimization for the Indonesian city of Padang. In Pedestrian and Evacuation Dynamics [1].

[42] C. Lieberman, D. Kurowski, M. Avila, L. Ricci, N. Thomas, C. Collyer, and B. Aguirre. Conceptual framework for simulating the pedestrian evacuation behavior from buildings. Paper 05-2297, Transportation Research Board Annual Meeting, Washington, D.C., 2005.

[43] E. Lim and B. Wolshon. Modeling and performance assessment of contraflow evacation termination points. Transportation Research Record, 1922:118-128, 2005.

[44] Q. Lu, B. George, and S. Shekhar. Capacity constrained routing algorithms for evacuation planning: A summary of results. LNCS, 3633:291-307, 2005.

[45] MATSIM www page. MultiAgent Transport SIMulation. http://matsim.org/, accessed 2008. 
[46] J. McCloskey, A. Antonioli, A. Piatanesi, K. Sieh, S. Steacy, S. Nalbant, M. Cocco, C. Giunchi, J. Huang, and P. Dunlop. Tsunami threat in the indian ocean from a future megathrust earthquake west of Sumatra. Earth and Planetary Science Letters, 265:61-81, 2008.

[47] S. Meyers. Effective STL: 50 specific ways to improve the use of the Standard Template Library. Addison-Wesley, 2002.

[48] Y. Murakami, T. Ishida, T. Kawasoe, and R. Hishiyama. Scenario description for multi-agent simulation. In Autonomous agents and multiagent systems (AAMAS'03), Melbourne, Australia, July 2003.

[49] D. Natawidjaja, K.Sieh, M. Chlieh, J. Galetzka, B. Suwargadi, H. Cheng, R. Edwards, J.-P. Avouac, and S. Ward. Source parameters of the great Sumatran megathrust earthquakes of 1797 and 1833 inferred from coral microatolls. Journal of Geophysical Research, 111, 2006.

[50] K. Nishinari, A. Kirchner, A. Nazami, and A. Schadschneider. Extended floor field CA model for evacuation dynamics. IEICE Transactions on Information and Systems, E87-D(3):726-732, 2004.

[51] Oak Ridge Evacuation Modeling System, accessed 2006. wwwcta.ornl.gov/cta/One_Pagers/OREMS.pdf.

[52] J. d. D. Ortúzar and L. Willumsen. Modelling transport. Wiley, Chichester, 1995.

[53] S. Pallottino and M. Scutella. Shortest path algorithms in transportation models: Classical and innovative aspects. In P. Marcotte and S. Nguyen, editors, Equillibrium and Adavanced Transportation Modelling, pages 245281. Kluwer, 1998.

[54] X. Pan, C. Han, K. Dauber, and K. Law. A multi-agent based framework for the simulation of human and social behavior during emergency evacuations. AI and Society, 22(2):113-132, 2007.

[55] W. Predtetschenski and A. Milinski. Planning for Foot Traffic in Buildings. Amerind Publishing Co. Pvt. Ltd., New Delhi, 1978.

[56] C. Rogsch. Vergleichende Untersuchungen zur Simulation von Personentrömen. Diploma thesis, Universität Wuppertal, 2005.

[57] P. Sattayhatewa and B. Ran. Developing a dynamic traffic management model for nuclear power plant evacuation. Paper, Transportation Research Board Annual Meeting, Washington, D.C., 2000.

[58] H. Sbayti, C.-C. Lu, and H. Mahmassani. Efficient implementation of method of successive averages in simulation-based dynamic traffic assignment models for large-scale network applications. Transportation Research Record, (2029):22-30, 2007. 
[59] V. Schneider and R. Könnecke. Simulating evacuation processes with ASERI. In Schreckenberg and Sharma [60], pages 303-314.

[60] M. Schreckenberg and S. D. Sharma, editors. Pedestrian and Evacation Dynamics. Proceedings of the 1st international conference, Duisburg, 2001. Springer, 2002.

[61] Y. Sheffi. Urban Transportation Networks: Equilibrium Analysis with Mathematical Programming Methods. Prentice-Hall, Englewood Cliffs, NJ, USA, 1985.

[62] P. Simon, J. Esser, and K. Nagel. Simple queueing model applied to the city of Portland. International Journal of Modern Physics C, 10(5):941960, 1999.

[63] R. Standish. Classdesc Library, accessed 2005. See parallel.hpc.unsw.edu.au/rks/classdesc.

[64] D. Strippgen and K. Nagel. Using common graphics hardware for multiagent simulation with CUDA. In Proceedings of the 2 nd International Conference on Simulation Tools and Techniques, to appear.

[65] H. Taubenböck, J. Prost, R. Kiefl, A. Roth, F. A. Ismail, G. Strunz, and S. Dech. Risk and vulnerability assessment to tsunami hazard using very high resolution satellite data. In C. Jürgens, editor, Remote Sensing - New Challenges of High Resolution, Bochum, 2008. European Association of Remote Sensing Laboratories.

[66] H. Taubenböck and A. Roth. A transferable and stable classification approach in various urban areas and various high resolution sensors. In Urban Remote Sensing Joint Event, Paris, 2007.

[67] G. Theodoulou and B. Wolshon. Alternative methods to increase the effectiveness of freeway contraflow evacation. Transportation Research Record, 1865:48-56, 2004.

[68] U. Weidmann. Transporttechnik der Fussgänger, volume 90 of Schriftenreihe des IVT. Institute for Transport Planning and Systems ETH Zürich, 2 edition, 1993. In German.

[69] Y. Wen, R. Balakrishna, M. Ben-Akiva, and S. Smith. Online deployment of dynamic traffic assignment: architecture and run-time management. In Intelligent Transport Systems, IEE Proceedings, volume 153, pages 76-84, 2006. 